\title{
Corrigendum
}

\section{Effect of Hypertension on Childhood-onset Systemic Lupus Erythematous in a Tertiary Medical Center in Korea}

\author{
Jeong Yeon Kim, M.D., Heeyeon Cho, M.D., Ph.D. \\ Department of Pediatrics, Samsung Medical Center, Sungkyunkwan University School of Medicine, Seoul, South Korea
}

Child Kidney Dis 2020;24:107-114

DOl: https://doi.org/10.3339/jkspn.2020.24.2.107

\section{Follow-up BP trends}

During follow up, the prevalence of hypertension increased and at the last visit, $31.3 \%$ of patients were diagnosed as having hypertension ( $\mathrm{n}=10)$. In the last follow-up, stage 2 hypertension was prevalent than stage 1 . The median value of Ped-SDI at last follow up was 1.0. Among childhood-onset SLE patients with hypertension, 2 patients had 3 episodes of posterior reversible encephalopathy syndrome. LVH was detected in 5 patients with hypertension and 1 patient without hypertension (Table 6).

Table 6. Follow-up Blood Pressure Trends

\begin{tabular}{lc}
\hline Variable & Values \\
\hline Last visit SBP (mmHg), mean \pm standard deviation & $116.0 \pm 13.4$ \\
Last visit DBP (mmHg), mean \pm standard deviation & $71.1 \pm 11.9$ \\
$\begin{array}{l}\text { Last follow up blood pressure stage, N (\%) [number } \\
\text { of patients with antihypertensive medication] }\end{array}$ & \\
Normotensive & $16(51.6)[2]$ \\
Elevated blood pressure & $12(38.7)[5]$ \\
Hypertension stage 1 & $1(3.2)[0]$ \\
Hypertension stage 2 & $2(6.5)[2]$ \\
Last visit BMI, median (range) & $21.2(15.8-31.8)$ \\
Last visit overweight and obesity, N (\%) & $11(35.5)$ \\
Last steroid dose, converted to PD (mg/kg/day), & $0.1(0.03-0.6)$ \\
median (range) & \\
LVH at any period*, N (\%) & $6(42.9)$ \\
PRES, N (\%) & $2(6.3)$ \\
\hline Last visit Ped-SDI, median (range), N (\%) & $1.0(0-5)$ \\
\hline
\end{tabular}

*In 14 patients, data was available.

Abbreviations: SBP, systolic blood pressure; DBP, diastolic blood pressure; $\mathrm{BMI}$, body mass index; PD, prednisolone; LVH, left ventricular hypertrophy; PRES, posterior reversible encephalopathy syndrome; Ped-SDI, The Pediatric Systemic Lupus International Collaborating Clinics/American College of Rheumatology Damage Index.

This is an open-access article distributed under the terms of the Creative Commons Attribution Non-Commercial License (http:// creativecommons.org/licenses/by-nc/4.0/) which permits unrestricted non-commercial use, distribution, and reproduction in any medium, provided the original work is properly cited. 